\title{
Psicologia e eduCaÇão em Periódicos brasileiros anterioRes a 1962
}

\author{
Mitsuko Aparecida Makino Antunes ${ }^{1}$
}

( $)$ presente trabalho tem como objetivo apresentar os resultados de parte de uma pesquisa ${ }^{2}$ produzida no Programa de Estudos Pós-graduados em Psicologia da Educação da Pontifícia Universidade Católica de São Paulo - PUCSP, cuja finalidade foi a de contribuir para a compreensão das relações que se estabeleceram entre Psicologia e Educação no Brasil, no período que vai de 1930 à regulamentação da profissão de psicólogo (1962). Mais especificamente, este trabalho trata das publicações nos principais periódicos de Psicologia e de Educação, compreendendo o período que vai de 1944 (data da publicação do primeiro número da Revista Brasileira de Estudos Pedagógicos - RBEP) até 1962 (data - limite deste estudo). Pretende-se aqui apresentar um estudo quantitativo dos dados obtidos, cuja finalidade é demonstrar as tendências gerais que caracterizam a produção em foco.

Este estudo faz parte de um projeto mais amplo, que visa a uma sistematização da história da Psicologia no Brasil, enfocando particularmente os anos que vão da década de 1930 à Lei 4119/62, considerando que esse período $^{3}$ tem carecido de síntese, tal como já se tem disponível para períodos anteriores ${ }^{4}$. Isso se deve ao fato de que a quantidade, a diversidade e a amplitude das produções nesse momento histórico exigem estudos mais extensos, não sendo possível dar conta de sua complexidade num único projeto de pesquisa, além do que a envergadura da tarefa não possibilita que ela seja empreendida por um pesquisador solitário. Faz-se necessário empreender pesquisas mais pontuais, abordando facetas específicas do período, cuidando de priorizar as questões que são mais fundamentais. Nesse sentido, as articulações entre Psicologia e Educação constituemse em elementos de fundamental importância para a compreensão desse período.
Considerando o período anterior, pode-se dizer que tal relação foi essencial para o processo de autonomização da Psicologia no Brasil, de tal maneira que se pode dizer que a Educação foi o terreno fértil no qual a Psicologia se desenvolveu. Essa relação permaneceu forte nos anos subseqüentes, podendo-se dizer que foi no campo da Educação que a Psicologia mais efetivamente encontrou as bases para seu desenvolvimento e, ao mesmo tempo, que foi na Psicologia que mais o campo da Educação fundamentou suas teorias e práticas. Dando seqüência ao processo de reconhecimento da autonomia da Psicologia como área específica do saber, segue-se um período no qual ela se consolida, ampliando sua produção nas esferas do ensino, da pesquisa e da prática, gerando as condições para o reconhecimento legal da profissão de psicólogo.

Assim, faz-se necessário o empreendimento de pesquisas que procurem demonstrar mais analiticamente a natureza dessas relações, como esforço para compreender o processo histórico de construção da Psicologia no Brasil e, concomitantemente, as características específicas que tal relação produziu no âmbito da educação brasileira. Este trabalho pretende contribuir para a compreensão desse período por meio do estudo de publicações em periódicos, considerando que esses são privilegiados meios pelos quais se expressam as principais produções da área.

\section{MÉTODO}

Foram escolhidos periódicos de Psicologia e de Educação, a saber: Revista Brasileira de Estudos Pedagógicos; Boletim e Revista de Psicologia Normal e Patológica; Arquivos Brasileiros de Psicotécnica; Boletim

\footnotetext{
${ }^{1}$ Professora do Programa de Estudos Pós-graduados em Psicologia da Educação da Pontifícia Universidade Católica de São Paulo - PUCSP. ${ }^{2}$ Essa pesquisa foi denominada Psicologia e Educação no Brasil: leitura histórica (1930-1962), da qual participaram como colaboradores: Aliciene Fusca Machado; Ana Cristina Arzabi; Isani Pereira da Silva; Jane Persinotti Trujillo; Lélia Carrasco Buscañan; Lilia Midori Shimizu P. dos Santos; Lina Maria de M. Carvalho; Maria de Fátima F. de O. Peruchi; Pedro Adilson da Rocha; Rita de Cássia Maskell Rapold; Silvia Mendes Pessoa e Solange Leme de Oliveira.

${ }_{3}$ À guisa de periodização, algumas características, tomadas como um todo coerente, podem justificar os seguintes períodos na História das Idéias Psicológicas e da Psicologia no Brasil: 1. pré-institucional (Pessotti, 1988); 2. institucional (Pessotti,1988); 3. autonomização; 4. consolidação e 5. profissional; entretanto, dadas as transformações ocorridas no âmbito da ciência e da profissão, é possível afirmar que estejamos já num novo período histórico, caracterizado pela extensão da Psicologia a um espectro mais amplo da vida social brasileira, marcado pelo gradativo compromisso social.

${ }^{4}$ Uma síntese do período colonial e do século XIX pode ser encontrada em: Massimi, M. (1990). História da Psicologia Brasileira., São Paulo, EPU. Uma síntese do processo de autonomização da Psicologia no Brasil pode ser encontrada em: Antunes, M. A. M. (1998). A Psicologia no Brasil: leitura histórica de sua constituição. São Paulo, EDUC e UNIMARCO.
} 
de Psicologia; Boletim da Faculdade de Filosofia, Ciências e Letras da USP, particularmente os números especiais das cátedras de Psicologia e de Psicologia Educacional; Revista de Pedagogia e Planejamento e Pesquisa. Essa escolha deveu-se ao fato de que esses periódicos constituíam-se nos principais meios de difusão do conhecimento produzido nas respectivas áreas.

Cada uma das publicações, no período especificado, foi exaustivamente examinada por grupos de pelo menos dois pesquisadores trabalhando independentemente, sendo destacadas as publicações que se enquadrassem nos critérios previamente estabelecidos (assunto relativo à Psicologia em periódicos de Educação e assunto relativo à Educação em periódicos de Psicologia). Em seguida, os resumos dos artigos, quando existiam, foram lidos pelo pequeno grupo e seus conteúdos discutidos por todos os pesquisadores, com a finalidade de refinar o critério de inclusão ou não de cada publicação como sendo de Psicologia e Educação concomitantemente; quando não havia resumo, era realizada uma leitura ainda assistemática e, em seguida, procedia-se como acima citado.

Após o levantamento dos artigos, foram elaborados quadros em ordem cronológica por periódico e, posteriormente, por categorias elaboradas a posteriori, referentes às temáticas específicas tratadas. Foram encontradas publicações que podem ser caracterizadas como: artigo, relato de pesquisa, relato de experiência, ensaio, resenha, notícia (sobre congressos, por exemplo). Eis alguns dos temas abordados nas publicações: orientação educacional/profissional; psicologia, educação e trabalho (muitas vezes relacionados à orientação educacional/profissional); problemas, distúrbios, dificuldades escolares; psicometria; educação especial; formação de educadores; educação e cidadania; regulamentação da profissão e formação de psicólogos, quando referentes ao ensino de psicologia; teorias e pesquisas sobre desenvolvimento, aprendizagem e outros com menor incidência. Para a análise foram excluídos os itens referentes a resenhas e notícias, pois, em função do significativo número de itens encontrados, a grande maioria na Revista de Psicologia Normal e Patológica, do Instituto de Psicologia da PUC-SP, sua freqüência poderia afetar a análise, uma vez que aqui se pretende uma abordagem mais quantitativa.

\section{Resultados}

Com base nos critérios citados, foram identificadas 200 publicações, sendo 100 em periódicos de psicologia e 100 em periódicos de educação ${ }^{5}$. Merece destaque o fato de que é significativo o número de Publicações referentes à Psicologia e Educação nos periódicos estudados, se comparado a outros campos de ação na Psicologia (em periódicos desta área) ou a outras perspectivas de enfoque educacional (em periódicos de Educação). Em outra pesquisa ${ }^{6}$, que se ocupou de registrar as realizações da Psicologia em obras de história da Psicologia no Brasil, também o campo da Educação aparece como o mais freqüente, apenas ultrapassado pelas referências de ordem geral, que não pertenciam a uma área ou campo de ação específico. Segue, na Tabela 1, a distribuição de publicações por periódico, subdivididos em periódicos de Psicologia e de Educação.

Tabela 1: Freqüência de publicações por periódicos de Psicologia e de educação.

\begin{tabular}{lcc}
\hline REVISTA & Número de publicações & Porcentagem \\
\hline ARQUIVOS (Psic.) & 34 & 17.0 \\
BOLETIM (Psic.) & 30 & 15.0 \\
FFCL USP (Psic.) & 08 & 4.0 \\
RPNP (Psic.) & 28 & 14.0 \\
SUBTOTAL PSIC. & 100 & 50.0 \\
PESQ-PLAN (Educ.) & 04 & 2.0 \\
REV-PED (Educ.) & 07 & 3.5 \\
RBEP(Educ.) & 89 & 44.5 \\
SUBTOTAL EDUC. & 100 & 50.0 \\
TOTAL & 200 & $\mathbf{1 0 0 . 0}$ \\
\hline
\end{tabular}

\section{LEGENDA:}

ARQUIVOS (Arquivos Brasileiros de Psicotécnica)

BOLETIM (Boletim de Psicologia)

FFCL USP (Boletim da Faculdade de Filosofia, Ciências e Letras da USP)

RPNP (Revista de Psicologia Normal e Patológica/Instituto de Psicologia da PUC-SP)

PESQ-PLAN (Pesquisa e Planejamento)

REV-PED (Revista de Pedagogia)

RBEP (Revista Brasileira de Estudos Pedagógicos)

Psico (Periódico de Psicologia)

Educ. (Periódico de Educação)

Percebe-se pela Tabela 1 que, apesar do número de publicações em periódicos de Psicologia e de Educação serem idênticas, sua distribuição é heterogênea. Isso não

\footnotetext{
${ }^{5}$ É preciso sublinhar que os números encontrados devem-se exclusivamente ao critério adotado para seleção e inclusão das publicações, não tendo havido nenhuma tentativa de "arredondá-los".

${ }^{6}$ Antunes, M.A.M. e colaboradores. A consolidação da Psicologia no Brasil (1930-1962): sistematização de dados e primeiras aproximações analíticas. Inédito.
} 
se aplica aos periódicos de Psicologia, pois o Boletim da Faculdade de Filosofia, Ciências e Letras da USP (FFCL-USP), que aparece com 8 publicações, pertencia à faculdade como um todo, tendo dedicado alguns números específicos às cátedras de Psicologia e de Psicologia Educacional, o que justifica a diferença em relação aos outros periódicos que, por sua vez, apresentam freqüências bastante próximas. A discrepância é encontrada nos periódicos de Educação, em que a Revista Brasileira de Estudos Pedagógicos (RBEP) é responsável por $89 \%$ das publicações. Devese ressaltar que a RBEP, o mais antigo dos periódicos estudados (o primeiro número foi publicado em 1944), foi e continua sendo um dos mais importantes periódicos de Educação, além de ser expressão editorial do Instituto Nacional de Estudos Pedagógicos - INEP, instituição que esteve intimamente ligada a projetos educacionais que tinham na Psicologia um de seus principais sustentáculos científicos, sob a influência de pioneiros da Psicologia no Brasil, como Lourenço Filho e Isaías Alves, dentre outros. Observa-se que a RBEP constituiuse num dos principais canais para a difusão da Psicologia da Educação, excedendo em número de publicações todos os periódicos específicos de Psicologia. Esses dados vêm confirmar análises anteriores que afirmam ter sido a Educação o principal campo no interior do qual a Psicologia se desenvolveu e se consolidou no Brasil $^{7}$, assim como demonstram as questões que estavam em pauta e que caracterizaram aquele momento específico.

Em função da própria temática em foco neste trabalho - Psicologia e Educação - considerou-se que seria relevante averiguar a incidência de referências à criança e à adolescência, uma vez que esses temas são recorrentes na Psicologia da Educação e fontes de algumas de suas principais demandas. Seguem os dados relativos às publicações que se referem explicitamente à infância ou sugerem esse enfoque (relacionados à escola primária, por exemplo), o mesmo em relação à adolescência e outros que são de caráter geral, para fins de comparação.

A Tabela 2 demonstra que a preocupação com a criança é recorrente nas publicações de Psicologia e Educação, totalizando 102 referências num total de 204, isso é, exatamente $50 \%$. Reitera-se, com esse dado, que a preocupação com aspectos da Psicologia Infantil é a principal temática do que podemos considerar Psicologia da Educação, o que confirma a manutenção da tendência dos períodos anteriores ao estudado e, sobretudo, sua permanência na atualidade.

As publicações sobre crianças são encontradas sob diferentes perspectivas. Há enfoques genéricos, como Aspectos da psicologia infantil, de Medeiros (RBEP,1949); questões relacionadas à linguagem e ao desenho, como Assuntos predominantes da linguagem do pré-escolar, de Marinho (RBEP, 1944) e Psicologia do desenho infantil, de Katzenstein (Boletim de Psicologia, 1954); desenvolvimento, tal como Teste de desenvolvimento motor: pesquisa com 100 crianças, publicada por Brasiliense (RPNP, 1958); há significativo número de artigos abordando "problemas escolares", dentre eles, Crianças que não aprendem, de Santos (RBEP, 1949); publicações abordando comportamento e conduta infantil, assim como os fatores que os influen-

Tabela 2: Freqüência de publicações sobre infância, sugestivos de infância, adolescência, sugestivos de adolescência e gerais

\begin{tabular}{|c|c|c|c|c|c|}
\hline Periódico & Infância & Sugestivo de Infância & Adolescência & Sugestivo de Adolescência & Gerais \\
\hline ARQUIVOS & 02 & 07 & 05 & 02 & 18 \\
\hline BOLETIM & 10 & 07 & 01 & 01 & 11 \\
\hline FFCL-USP & 02 & 01 & 01 & 01 & 03 \\
\hline RPNP & 10 & 06 & 01 & 00 & 12 \\
\hline TOTAL PSIC. & 24 & 21 & 08 & 04 & 44 \\
\hline PESQ-PLAN & 02 & 02 & 00 & 00 & 00 \\
\hline REV-PED & 03 & 03 & 01 & 00 & 00 \\
\hline RBEP & 29 & 18 & 05 & 03 & 37 \\
\hline TOTAL EDUC. & 34 & 23 & 06 & 03 & 37 \\
\hline TOTAL & 58 & 44 & 14 & 07 & 81 \\
\hline
\end{tabular}

Obs.: o número de referências excede o número de publicações, pois alguns artigos referem-se a duas categorias concomitantemente, como "Tarefas evolutivas das crianças e adolescentes", por exemplo. 
ciam, como A criança e o cinema, de Pfromm Neto (Boletim de Psicologia, 1959); encontram-se também publicações de natureza teórica ou mesmo de história da psicologia, como Reflexões sobre meio-século da psicologia da criança (a evolução histórica das idéias sobre desenvolvimento infantil - suas relações teóricas), de Zazzo (RBEP, 1956).

O enfoque sobre adolescentes, embora apareça numa frequiência bem menor, traz temas relacionados à necessidade de conhecimento sobre os processos que envolvem esse período do desenvolvimento e suas relações com a prática pedagógica, sugerindo sua especificidade no âmbito da educação. Dentre as publicações, podemos citar: Características gerais da adolescência, de Camargo e Garcia (Boletim de Psicologia, 1956); Adolescentes no tempo atual, de Katzenstein (Boletim de Psicologia, 1957) e Mudar a atitude do educador diante do jovem, de Cardoso (RBEP, 1958).

Os temas gerais são bastante heterogêneos, mas devem ser destacados os inúmeros textos que tratam de questões eminentemente teóricas. Só a título de ilus- cional e/ou profissional. Cabe ressaltar que tais temas não se constituem na totalidade do que apareceu no levantamento, pois é ampla a variedade de assuntos; as categorias acima descritas foram selecionadas para análise em função de sua incidência, por caracterizar tendências que eram recorrentes naquele momento ou por demonstrar preocupações que permaneceram ao longo do tempo. Particularmente deve-se justificar a presença da categoria "orientação educacional/profissional”, pois esse campo de ação foi um dos que mais incorporaram a ciência psicológica a uma prática educativa então recente, tendo sido uma das mais importantes bases para o desenvolvimento da Psicologia Educacional e ponte para o estabelecimento de relações entre a Psicologia e o campo da organização e racionalização do trabalho; o mesmo pode ser dito em relação à categoria "problemas escolares", base também para a extensão da Psicologia da Educação para uma prática de natureza psicoterapêutica.

A Tabela 3 demonstra, antes de mais nada, uma distribuição muito semelhante entre os periódicos de

Tabela 3: Distribuição de publicações por assunto

\begin{tabular}{|c|c|c|c|c|c|c|c|}
\hline Periódico & Desenvolvimento & Aprendizagem & $\begin{array}{l}\text { Comportamento, } \\
\text { conduta, atitude }\end{array}$ & Psicometria & $\begin{array}{l}\text { Problemas } \\
\text { Escolares }\end{array}$ & $\begin{array}{l}\text { Educação } \\
\text { Especial }\end{array}$ & Orientação \\
\hline ARQUIVOS & 00 & 07 & 09 & 07 & 05 & 00 & 14 \\
\hline BOLETIM & 13 & 06 & 04 & 05 & 03 & 00 & 04 \\
\hline FFCL-USP & 01 & 01 & 04 & 02 & 01 & 01 & 01 \\
\hline RPNP & 01 & 00 & 10 & 06 & 07 & 05 & 07 \\
\hline TOTAL PSIC. & 15 & 14 & 27 & 20 & 16 & 06 & 26 \\
\hline PESQ-PLAN & 01 & 01 & 01 & 00 & 00 & 00 & 00 \\
\hline REV-PED & 00 & 03 & 01 & 01 & 02 & 01 & 01 \\
\hline RBEP & 13 & 11 & 25 & 19 & 18 & 07 & 14 \\
\hline TOTAL EDUC. & 14 & 15 & 27 & 20 & 20 & 08 & 15 \\
\hline TOTAL & 29 & 29 & 54 & 40 & 36 & 14 & 41 \\
\hline
\end{tabular}

Obs:- as categorias não são mutuamente exclusivas, pois várias publicações ajustam-se concomitantemente em diferentes categorias.

tração, pode ser citado o artigo: $O$ sentido atual da Psicologia Educacional, de Angelini (RPNP, 1962).

Os dados sugerem várias temáticas ou abordagens presentes nas publicações, revelando um amplo espectro de produções, dentre as quais destacamos algumas que são recorrentes nos vários periódicos. A Tabela 3 apresenta a distribuição de publicações nos periódicos, referentes aos seguintes assuntos: desenvolvimento; aprendizagem; comportamento, conduta, atitudes; psicometria; problemas (dificuldades, disfunções, distúrbios) escolares; educação especial e orientação educa-
Educação e de Psicologia, sugerindo que não havia uma linha de demarcação temática entre as publicações de ambas as áreas. É necessário reiterar que, nesse momento, dando prosseguimento à tendência do período anterior, a Educação continuava sendo o principal terreno sobre o qual a Psicologia se desenvolvia e se consolidava no país, ao mesmo tempo que era preponderante a presença dessa ciência no âmbito da Educação, haja vista a quantidade de publicações na RBEP.

$\mathrm{O}$ maior índice obtido na Tabela 3 refere-se à categoria “comportamento, conduta e atitudes", embora isso 
se explique pelo fato de que aqui são inseridas publicações de caráter geral ou que podem ser consideradas como pertinentes àquilo que se denomina Psicologia geral, o que justificaria a alta freqüência. Foram encontrados artigos referentes aos seguintes temas: personalidade; diferenças individuais; relações interpessoais; relações professor-aluno; relações família-escola; motivação; aptidão; interesses vocacionais; adaptabilidade e ajustamento; disciplina; liderança; conceitos morais; formação de atitudes democráticas e educação democrática; fatores etno-psicológicos; influências sobre o comportamento e a conduta; preconceito; questões de ordem afetivo-emocional, como: ansiedade, agressividade, cólera, medo, insegurança e angústia (conteúdos estes que serão trabalhados mais especificamente em "problemas escolares").

De um lado, são explícitas as tendências próprias da época, como a preocupação com diferenças individuais, motivação, aptidão e vocação, sobretudo relacionadas à orientação educacional/profissional e à utilização de testes psicológicos. Esses dados refletem também as demandas de uma sociedade em busca de sua inserção no mundo industrializado, projeto este que teve na articulação entre Educação e Psicologia uma importante contribuição para a realização de seus fins, especialmente no que diz respeito aos métodos de racionalização do trabalho, representado, entre outras formas de intervenção, na adequação do trabalhador à tarefa, por meio da orientação educacional/profissional aos jovens aprendizes. O Serviço Nacional de Aprendizagem Industrial SENAI (como instituição formadora de mão-de-obra) e o Instituto de Seleção e Orientação Profissional da Fundação Getúlio Vargas - ISOP/FGV (como instituição produtora de pesquisas, projetos de intervenção e assessoria com base na Psicologia e responsável pela publicação dos Arquivos Brasileiros de Psicotécnica) foram significativos produtores de conhecimento e prática nesse âmbito, representados aqui por publicações em diferentes periódicos. Esses dados reforçam a análise que indica a Educação como uma das principais bases para a estruturação do campo da Psicologia aplicada à organização do trabalho. Além disso, as publicações sobre orientação educacional/profissional aparecem como a segunda categoria mais freqüente, reforçando a análise acima.

Entretanto, muitos dos trabalhos publicados tratam de assuntos que permanecem até hoje em pauta. Dentre eles, a preocupação com a construção de uma esco- la democrática e a busca de ações educativas para a formação de atitudes democráticas nos alunos, como se vê nos artigos de Antipoff (RBEP, 1944; 1945): Como pode a escola contribuir para a formação de atitudes democráticas? e Dos perfis caracterológicos como elementos para uma educação democrática. Encontram-se também artigos que abordam as relações interpessoais em educação, como: Educação e relações interpessoais, de Leite (Boletim de Psicologia, 1959); Influência da relação mestre-aluno no rendimento pedagógico, de Mira y Lopez (Arquivos, 1961); Relações entre família e escola na primeira infância, de Queiroz (RPNP, 1962) e Relações entre a escola e a comunidade, de Pereira (Revista de Pedagogia, 1956). Temas que se aproximam do que poderíamos hoje denominar Psicologia Social da Educação também aparecem, dentre eles, outro artigo de Leite (FFCLUSP, 1950), Preconceito racial e patriotismo em 6 livros didáticos primários brasileiros e Aspectos psicológicos da influência do cinema sobre a criança e o adolescente, de Azzi (RPNP, 1957).

Outra questão recorrente nos artigos estudados e foco bastante atual de preocupação diz respeito aos denominados "problemas de aprendizagem" (expressão esta que atribui mais diretamente o determinante à própria criança) ou, mais adequadamente, "problemas de escolaridade". Os títulos dos artigos expressam o que era considerado como "problema" e as formas de abordálo, seja em termos explicativos ou em âmbito de intervenção. O Quadro 1 contém artigos que ilustram os diferentes conteúdos tratados sob a rubrica "problemas".

Percebe-se, pelos artigos citados no Quadro 1, que a maioria enfoca questões relacionadas a comportamento, conduta e atitudes, alguns relacionados a elementos de natureza afetivo-emocional. Destacam-se expressões como: crianças difíceis, delinqüentes e distúrbios de comportamento; ansiedade, agressividade, cólera, medo e angústia; assim como a preocupação com o "ajustamento". Considerando que tais artigos aparecem indistintamente em periódicos de Educação ou de Psicologia, podese dizer que essa abordagem não era especificamente oriunda de preocupações mais estritamente psicológicas, mas consistiam também em demandas propriamente educacionais. Esses dados refletem a maneira como conteúdos da Psicologia foram incorporados pela Educação, muitos dos quais responsáveis por interpretações que serviriam para obscurecer os fatores propriamente escolares como determinantes de muitos dos problemas em 
Quadro 1: Publicações que tratam de "problemas escolares", em ordem cronológica

\begin{tabular}{|c|c|}
\hline ARTIGOS & AUTOR, PERIÓDICO, DATA \\
\hline $\begin{array}{l}\text { Aplicação dos métodos psicopedagógicos no estudo das fugas infantis } \\
\text { (comunicação ao } 18^{\circ} \text { Congresso Luso-Espanhol para o progresso nas ciências, } \\
\text { Córdova, 1944) }\end{array}$ & Maria I. L. da Costa RBEP, 1944 \\
\hline Dificuldades escolares e pedagogia clínica & Emile Planchard RBEP, 1945 \\
\hline Da clínica de leitura em um sistema escolar & Margareth Hall RBEP, 1947 \\
\hline Clínica para os defeitos da fala & Margareth Hall RBEP, 1947 \\
\hline Complexos Infantis & Maria I. L. da Costa RBEP, 1948 \\
\hline Problemas da adolescência: o querer valer, a evasão e o retorno & Ana Rimoli de Faria Doria RBEP, 1948 \\
\hline O diagnóstico nas crianças difíceis & Maria I. L. da Costa RBEP, 1949 \\
\hline Psicopatologia dos mitos e lendas dos contos infantis & Felix M. Ibanez RBEP, 1949 \\
\hline Crianças que não aprendem & Teobaldo M. Santos RBEP, 1949 \\
\hline Problemas das anormalidades no desenvolvimento psíquico & Werner Kssifer RBEP, 1951 \\
\hline Carência lúdica e escolaridade & Acrísio Cruz RBEP, 1951 rbep \\
\hline Ansiedade e agressividade em crianças pré-escolares. & Betti Katzenstein RBEP, 1953 \\
\hline Afeição, cólera e medo entre adolescentes estudantes da cidade de São Paulo & Maria José de Barros Fornari Aguirre FFCL, 1953 \\
\hline Ansiedade e agressividade em crianças pré-escolares & Betti Katzenstein RBEP, 1953 \\
\hline Dois casos de dificuldade na leitura e na escrita & Betti Katzenstein RBEP, 1954 \\
\hline $\begin{array}{l}\text { Maturidade, problemas relacionados à maturidade e o teste de } A B C \text { de } \\
\text { Lourenço Filho }\end{array}$ & Ofélia B. Cardoso RBEP, 1954 \\
\hline $\begin{array}{l}\text { A Dislexia e a Imaturidade de Trabalho, duas causas freqüentes de } \\
\text { reprovação no 1o grau da escola primária }\end{array}$ & Maria José de Barros Fornari Aguirre BOLETIM, 1956 \\
\hline A diagnose e a retificação da aprendizagem & Luiz Alves de Mattos REV-PED, 1956 \\
\hline Desacertos educacionais (Casos de orientação psicopedagógica no ISOP) & Maria Leite ARQUIVOS, 1957 \\
\hline Delinqüentes em potencial & Enzo Azzi ARQUIVOS, 1957 \\
\hline Problemas de ajustamento à escola & Elisa D. Veloso RBEP, 1958 \\
\hline Problemas atuais da adolescência & Maurice Herzog RBEP, 1959 \\
\hline Benefícios da hipnose em escolares desajustados & Jefferson Gonçalves Gonzaga RPNP, 1959 \\
\hline Dislexia & Jacyra Ismene Leite RPNP, 1960 \\
\hline A educação e o problema psicológico do medo e da angústia & Eliezer Schneider RBEP, 1960 \\
\hline Critérios para a análise dos distúrbios de comportamento na infância & Aidyl M. de Queiroz BOLETIM, 1961 \\
\hline Expressão gráfica de agressão & Anny Zausmer BOLETIM, 1961 \\
\hline
\end{tabular}

questão. Da mesma maneira, existem artigos que explicitam a abordagem clínica de tais "problemas" que, tal como na questão acima, também contribuíram para que a criança e não as condições escolares de ensinoaprendizagem e de relações interpessoais fossem consideradas como fatores importantes na determinação de muitos entraves encontrados no cotidiano escolar.

Menos freqüentes são os artigos relacionados às dificuldades de aprendizagem ou problemas de desenvolvimento, se considerados os temas acima discutidos. Dentre estes, as questões relacionadas à alfabetização parecem ser as fontes de maior interesse, ligadas muitas vezes à questão da maturidade; não pode ser esquecido o fato de que esse tema foi tratado pioneiramente por Lourenço Filho, personagem este que se constituiu num dos principais produtores, divulgadores e fomentadores da Psicologia no Brasil e, em especial, da Psicologia Educacional.

Voltando à Tabela 3, vê-se que a psicometria aparece com um número significativo de referências, confirmando uma tendência da época. Acrescente-se a isso que várias publicações em periódicos de educação (par- 
ticularmente a RBEP) foram excluídas deste estudo, por não serem explicitamente relacionadas àquilo que poderia ser considerado como Psicologia e Educação, embora pelo critério adotado (tema de Psicologia em periódico de Educação) elas estivessem inseridas no quadro original. A maioria dessas publicações refere-se a questões relativas à orientação profissional, envolvendo "seleção e treinamento" de trabalhadores por meio da utilização de testes psicológicos. A maioria dos artigos incluídos neste levantamento descreve pesquisas realizadas com testes psicológicos, tais como: Estudo sobre o teste de inteligência não-verbal de Pierre Weil aplicado em escolas particulares, de Schechtman (Arquivos, 1955); Rigidez e flexibilidade no teste de Rorschach de pré-escolares, de Scheffer (Arquivos, 1958); Um estudo do status mental de um grupo de crianças nordestinas de idade escolar, de Almeida (Boletim de Psicologia, 1959) e A criança de 7 anos através dos testes mentais aplicados em Belo Horizonte, de Lustosa (RBEP, 1945). Nota-se a variedade de tipos de testes estudados, incluindo inteligência, aptidão, interesse e testes projetivos, sobretudo o Rorschach.

Em educação especial são tratados temas como: ensino, psicopedagogia, ajustamento, educação e reeducação, orientação profissional, superdotados, deficientes físicos, surdos, portadores de deficiência mental e alguns casos pontuais de crianças acometidas por determinadas doenças. São também publicados artigos de autores estrangeiros e relatos de experiências estrangeiras em educação especial.

As publicações que tratam de desenvolvimento e aprendizagem, temas fundamentais da Psicologia da Educação, são em sua maioria relacionadas a pesquisas ou a discussões teóricas. Linguagem e vocabulário, desenho, maturação, aprendizagem intra-serial são alguns dos assuntos abordados nos artigos. Dentre estes, podemos citar: Experiência sobre seqüência de associações na aprendizagem intra-serial, de Santos e Ferreira (Arquivos, 1951); Contribuição ao estudo do material de pesquisas em aprendizagem verbal, de Angelini (Boletim de Psicologia, 1953) e Estudo e avaliação dos níveis de maturação, de Lourenço Filho (RBEP, 1952). Outros temas, em geral mais pontuais, são tratados nos artigos, cuja variedade e amplitude expressam até uma certa dispersão, mas demonstram que sob os rótulos de psicologia e educação houve uma significativa produção de conhecimento e práticas.

Outro elemento de análise é a freqüência de artigos de determinados autores. Arrigo Leonardo Angelini e Betti Katzenstein são os autores que mais publicaram. Devese dizer que o primeiro construiu sua carreira especificamente no âmbito da Psicologia Educacional, dedicandose à pesquisa e ao ensino (sucedeu Noemi Silveira Rudolfer na cátedra de Psicologia Educacional da Faculdade de Filosofia, Ciências e Letras da USP), o que justifica sua presença marcante na autoria das publicações que aqui são tratadas. Betti Katzenstein, por sua vez, não foi uma profissional dedicada exclusivamente a essa área, mas foi uma profícua produtora de pesquisas e publicações ao longo de sua carreira. Em seguida aparece Helena Antipoff que, como Angelini, dedicou-se exclusivamente à psicologia e à educação, porém, diferentemente dele, suas atividades estavam ligadas não apenas à pesquisa $\mathrm{e}$ ao ensino, mas sobretudo à prática educacional, tendo realizado inúmeras experiências educacionais em Minas Gerais, além de ter participado ativamente de várias realizações em âmbito nacional. Outros que aparecem com freqüência significativa são: Osvaldo de Barros Santos, Heloisa Marinho, Maria I. L. da Costa, Emilio Mira y Lopez, Margareth Hall e Dante Moreira Leite. Destaque deve ser dado a um tipo específico de produção, que não foi incorporado a este trabalho, geralmente de autoria de Enzo Azzi e Aniela Tadeusz-Ginsberg, relativo à publicação de resenhas de obras em psicologia e educação e notícias sobre congressos ocorridos no Brasil e, particularmente, em outros países.

Do ponto de vista institucional, os dados demonstram que foi a RBEP o mais produtivo periódico que se dedicou a publicar artigos relativos à articulação entre Psicologia e Educação. Ainda que tenha sido a RBEP o mais antigo periódico estudado, isso por si só não é suficiente para justificar a quantidade de sua produção; é mais provável a interpretação de que o projeto educacional assumido pelo Instituto Nacional de Estudos e Pesquisas Educacionais - INEP, órgão que ainda edita o referido periódico, tinha na Psicologia um de seus mais importantes pilares de sustentação, refletindo a tendência hegemônica da época, representada oficialmente por esse órgão, e tendo em seus quadros, dentre outros, Lourenço Filho, um dos mais profícuos pioneiros da Psicologia e, particularmente, da Psicologia Educacional no Brasil.

\section{Conclusão}

Algumas considerações podem ser feitas: não há diferença significativa entre os conteúdos das produções 
dos periódicos de Psicologia e de Educação; revelamse autores bastante produtivos; há heterogeneidade de referenciais teóricos; há diferentes concepções de educação e conseqüentemente diferentes formas de abordagem dessa questão; há temas tratados com originalidade e que são ainda atuais, assim como existem outros assuntos e abordagens que foram próprios da época e que são hoje considerados ultrapassados. Revela-se uma certa dicotomia entre autores/instituições produtores de pesquisa, de um lado, e aqueles voltados para a aplicação de outro; podem ser estabelecidas caracterizações institucionais, assim como alguns aspectos relativos à mudança de alguns enfoques ao longo do tempo.

Os dados e a análise ora apresentados referem-se a uma primeira aproximação com as publicações dos periódicos estudados, objetivando demonstrar as características mais gerais e as tendências que se apreendem a partir de uma abordagem mais quantitativa. Outras pesquisas estão sendo geradas a partir desses dados. Todas elas constituem-se em pesquisas qualitativas sobre os conteúdos dos artigos registrados.
Em geral, nessas pesquisas, tem-se confirmado aquilo que é aqui demonstrado, sobretudo em relação ao fato de que muitas idéias consideradas contemporâneas e inovadoras já eram defendidas há cerca de 50 anos, no Brasil, e por brasileiros; dentre estas, a defesa da educação inclusiva para as pessoas com deficiência; a necessidade de formação continuada na formação de professores; a crítica aos testes psicológicos como instrumentos de medida de determinadas funções específicas, desconsiderando as determinações de natureza socioeconômico e cultural sobre o sujeito. Entretanto, muitas das publicações revelam posições não apenas próprias da época, mas reveladoras das tendências em voga no momento. Há também abordagens hegemônicas no interior de algumas subáreas ou campos de atuação; divergências teóricas e relativas à prática psicoeducativa. Os dados revelam ainda a explicitação da consolidação da Psicologia no Brasil, condição para seu reconhecimento como profissão e, de certa maneira, definidora dos caminhos iniciais que a Psicologia profissional trilhou no país. 